\title{
Quality Control of Radiant Heaters
}

\author{
Daniel Aquilino González ${ }^{*}$ Francisco Javier Madruga, Maria Ángeles Quintela, \\ José Miguel López-Higuera \\ Photonics Engineering Group - Universidad de Cantabria, \\ Avda. Los Castros s/n 39005 Santander - SPAIN
}

\begin{abstract}
Based on infrared thermography, a non-destructive testing and evaluation (NDT\&E) procedure is proposed for defects assessment on radiant heaters. Under a short electrical excitation, an infrared camera captures the cooling process of the heaters. Breaking the thermographic images down not only makes easiest the location of defects but it also allows their classification. Several kinds of defects have been taken into account: lack of supporting brackets; defects originated by a deficiency in the heating material; those from an excess of heating material; and those parts of the heating elements which are in wrong contact (non-contact or semi-buried) with the substrate. Each kind of analyzed defect has a different thermal history after the electrical excitation because of its nature. By means of computer vision techniques, the defects can be spatially located. The "chain code" was employed to follow the pattern of the heating element and so concentrate the analysis in points belonging to the pattern. A good agreement with analysis made under human's criteria is achieved. However, using infrared cameras and processing the data with computer vision algorithms allows controlling in-site the quality of the product without any subjectivity. So, the heaters manufacturing industry could come along with the implementation of this automatic detection procedure. Experimental results that validate the proposed method will be presented and discussed in this paper.
\end{abstract}

Keywords: Thermography, Non-Destructive Evaluation and Testing, Quality control, Infrared technology, radiant heaters

\section{INTRODUCTION}

Nowadays, quality control has overcome one of the most important goals in any industry. Manufacturing has been relocated all around the world and competence has been strongly increased. In this scene, it is necessary to ensure the quality of the products to maintain some commercial advantage. Lot of money is therefore invested on processes whose main task is the detection of defects to eventually sort the products by its quality later.

Several Evaluation and Testing Techniques have been developed and improved during years helping the operator to decide whether defects are to be found or not. Each different technique has been optimized in its correspondent niches among the huge range of industrial processes and products available today. It is important to develop Non-Destructive Evaluation and Testing techniques (NDET) that are fast, non-contact and safe. These are the advantages of Infrared Thermography (IT) ${ }^{1}$ over other NDET techniques such as ultrasounds or X-rays.

In the case under study, the niche of application corresponds to radiant heaters. Manufacturing industries of these products are interested in control the appearance of defects on the heaters that could lead to a lack of effectiveness or even malfunction or breakdown. It is obvious that IT represents the easiest NDET technique to be applied to this quality control process. The functionality of the heater is derived from the aspect that the power dissipated in the resistor heats the resistor (Joule heating) and this heat is uniformly distributed. Therefore, under any excitation, the heating and cooling behaviors of any two parts of the heating wire should be the same. The energy radiated by a body in a particular spectral range is related to its temperature as it can be inferred from the Planck's law. This radiation can be detected by photodetectors or thermal detectors which are the base of infrared cameras. Consequently, the heaters could be perfectly tested by using an infrared camera when enough spatial resolution is provided.

\footnotetext{
"dagonfer@teisa.unican.es; Phone: +34-942-200877 ext.15; Fax: +34-942-200877; http://grupos.unican.es/gif
} 
Previous works have already given good results in this subject ${ }^{2}$. However, following studies have come upon some substantial improvements which are here presented. Previous inspections used to take several seconds and took advantage of a stationary state of the heater. The specimen was heated using an electrical control which supplied several cycles of heating and cooling periods. A ramp wave was induced with a maximum voltage of $150 \mathrm{~V}$ and equal rising and falling times of $10 \mathrm{sec}$ (duty cycle=50\%), Images were captured each $2 \mathrm{sec}$ and so the time of the whole recorded sequence was at least $20 \mathrm{sc}$.

Herein, a transient behavior of the heaters is analyzed. The time consumed for each inspection has now been strongly reduced. Based on a electronic timer, the excitation has been reduced to $0.1 \mathrm{sc}$, and it could even be shorter although it should always have enough energy to produce the heating. This quasi instantaneous pulse (ideally corresponds to a Dirac's Delta function) is used for the heating. Then the cooling is expected to take place by natural convection and radiance mechanisms. Both, the heating and the cooling tendency of the heater, are recorded by a faster infrared camera, compared with previous works, and a simple processing is applied to the images. All these efforts are focused on the development of a quasi real-time inspection process which could be embedded in a production line. Evaluation and testing of the product in situ, in the production line, would lead to the desired quality control for any manufacturer.

In this paper a description of the setup and the used processing technique for a quality control of radiant heaters is presented in the following section. Afterwards, the discussion of the images, provided by the software also developed specifically, leads to establish the pattern to identify the different defects in the heater. The performance in defect detection has been evaluated using commercial pieces containing a range of real and artificial defects.

\section{SETUP AND PROCESSING TECHNIQUE}

The setup in the lab can be observed in the Figure 1.

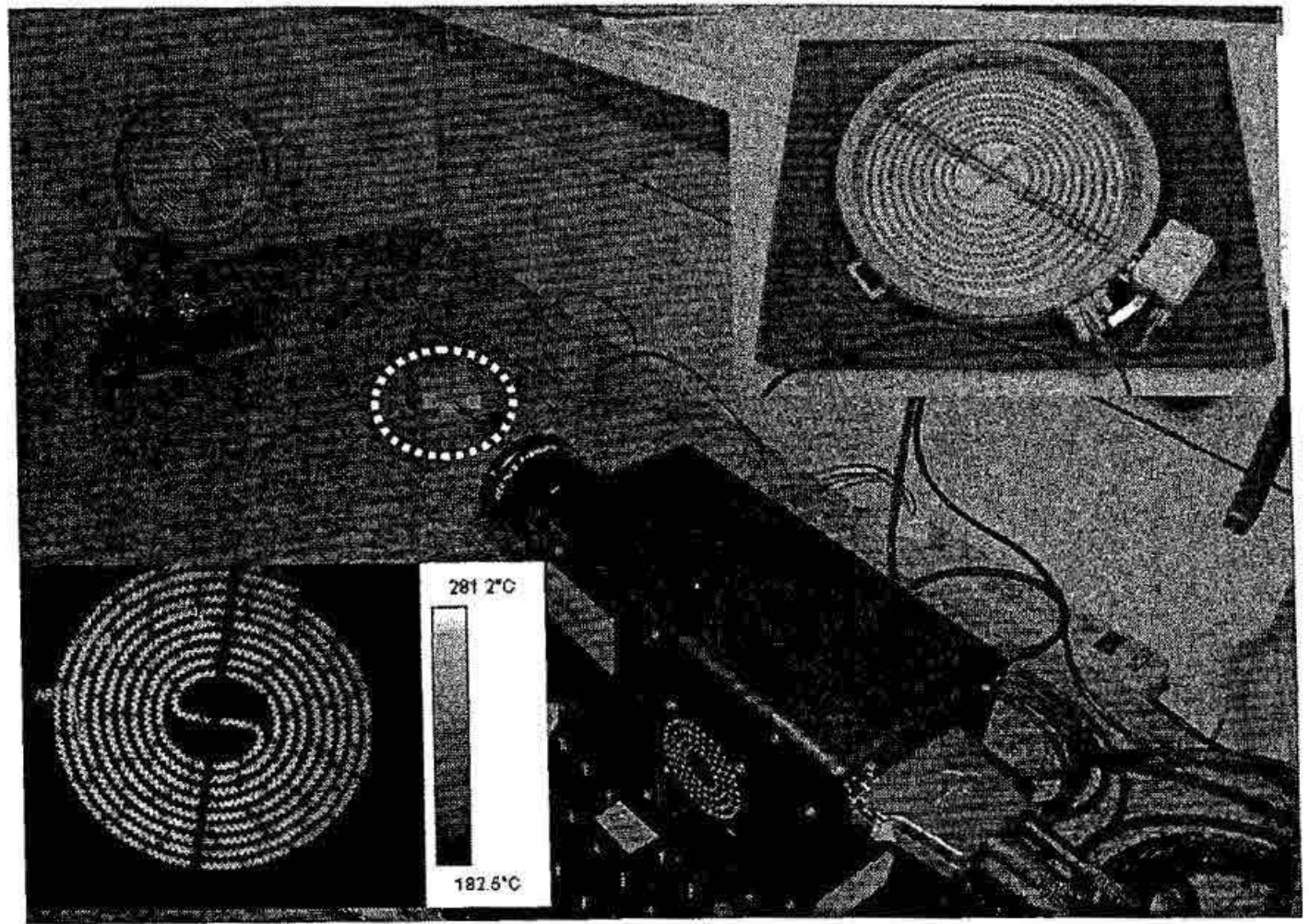

Figure 1. Setup used in the lab for the inspection $\mathrm{f}$ commercial radiant heaters. Within the image, a close-up of one of the heaters and a thermal image are provided. Surrounded by a circle, the electronic timer controls the duration of the excitation. 
Specimens were heated using an electrical control which offers a fast and precise energy shut off. Control on excitation is provided by a Crozet timer, in function $\mathrm{H}$, which energizes an output contact during a time that is adjustable. The interest was centered on short pulses as closer as possible $(0.1 \mathrm{sc})$ to the ideal Dirac's Delta function.

All the inspection, including the heating and cooling tendencies, is captured by an infrared camera. Working with transient timing of only $0.1 \mathrm{sc}$, fast cameras are needed to obtain several frames in such a heat transient. In this case, a CMT 128M Thermosensorik GmbH camera has been used due to its high frame rate and excellent temperature resolution $(\mathrm{NETD}<20 \mathrm{mK})$. Free sizeable subframes could be obtained up to a maximum subframe rate higher than $5 \mathrm{kHz}$.

The specimens for the inspection have been provided by a manufacturer of radiant heaters. These are commercial heaters with a range of real and artificial defects that could even been found in these products. Figure 2 shows some close-ups of the defects.

a)
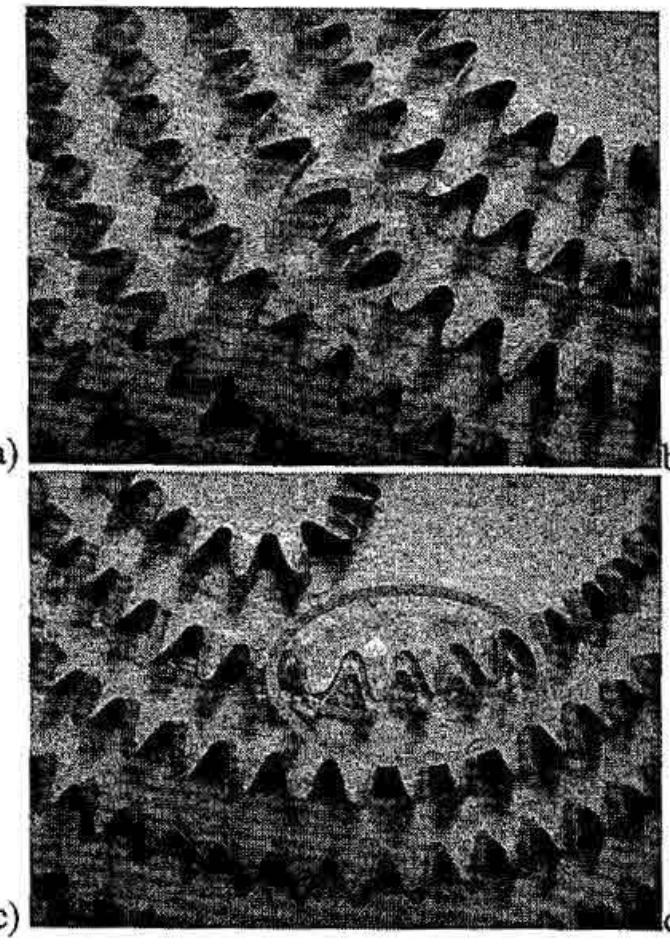

)
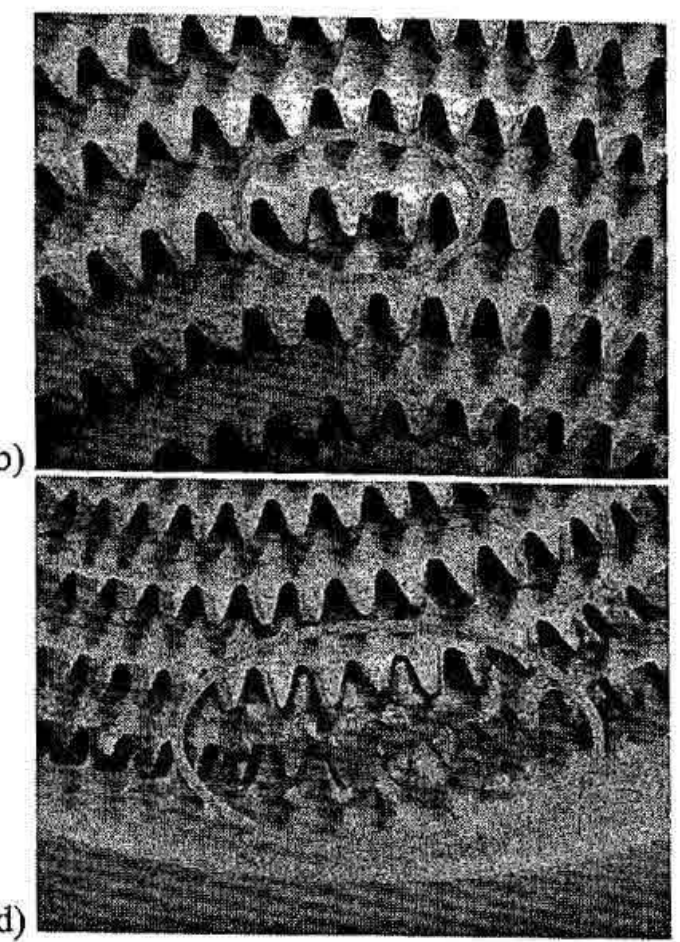

Figure 2, Real and artificial defects on radiant heaters: a) Excess of material as a consequence of a link. b) Lack of material as a consequence of a cut on the surface of the wire. c) and d) parts of the wire that are not in contact with the substrate.

\subsection{Processing}

Once the thermographic sequence has been captured, a simple algorithm has been developed to help in the detection and classification of defects. Its simplicity leads to a fast inspection, the goal desired for an online production quality control.

Comparing the heating and cooling tendencies for each pixel, we are able to detect those elements which exhibit a different way. Analyzing the heating and cooling tendencies, a classification of the defects can be carried out. Figure 3 illustrates the temporal evolution of several pixels. The difference in radiation intensity, captured by the camera for the pixels belonging to defective and non-defective areas, is shown. 

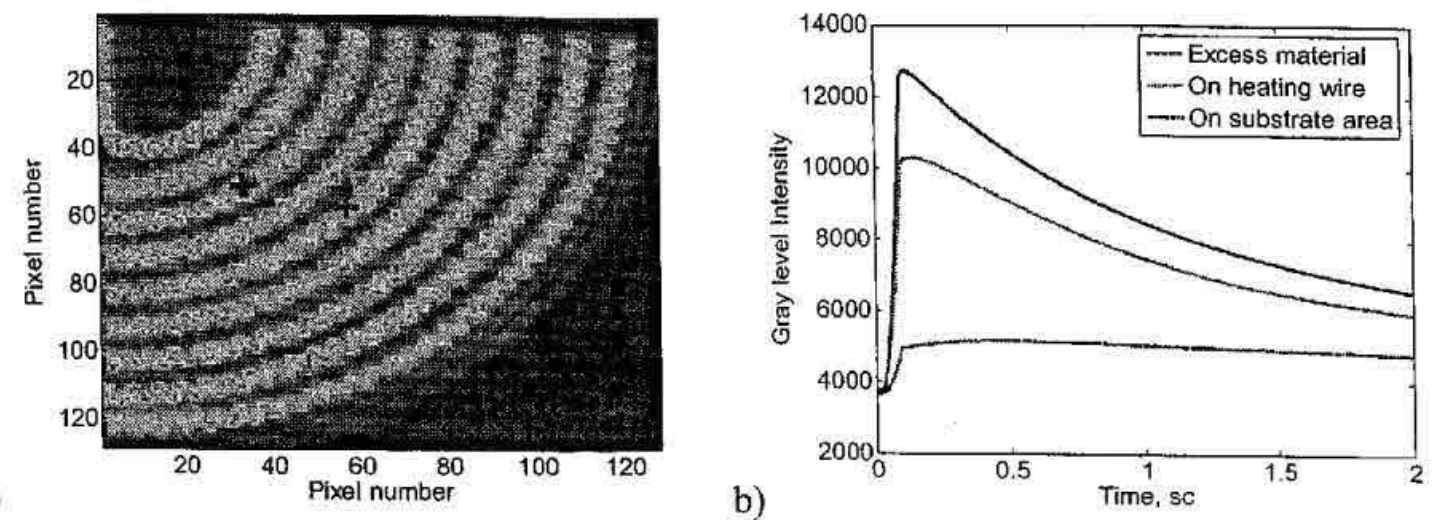

Figure 3. Temporal evolution of several pixels along the thermographic sequence. a) pixels on image b) evolution with obvious heating and cooling halves or flanks.

The detection of the defects could be achieved by simply comparing the intensities of the pixels. However, slight differences on surface emissivity (metals' emissivities depend strongly on the direction of the field of view) would give dissimilar intensities on pixels that could belong to the same non-defective area. Therefore, the analysis of the sequences must be performed over the tendencies instead of just observing the absolute value of the captured radiation intensity.

A code based on Matlab ${ }^{\mathrm{TM}}$ has been developed. It proceeds, as it is described below in next subsections, offering different images from the captured sequence.

\subsection{Heating process}

Each half (heating flank and cooling flank) can be fitted by a different tendency. For example, for the heating half, a quadratic fitting adjusts the trend of the intensity level as could be extracted from the Joule's effect. The rate at which heat is produced in a resistor is proportional to the square of the current flowing through it, if the resistance is constant (Joule's Law). Therefore, after the fitting, an image like the one displayed on Figure 4a representing the quadratic coefficient for each pixel leads to the classification of the defects as it is described below.

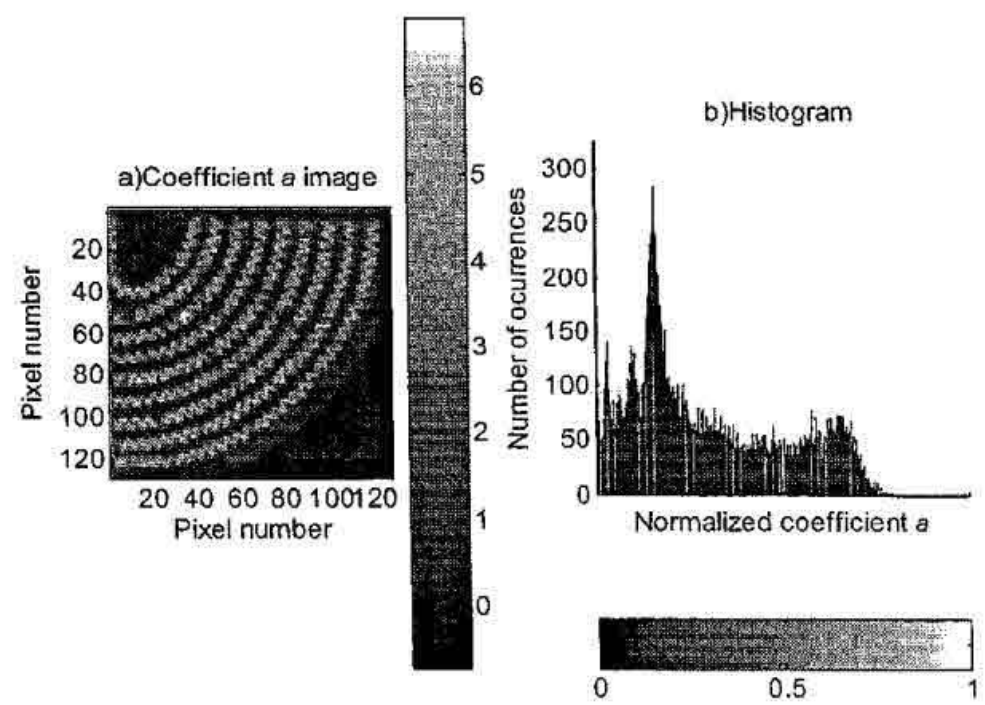

Figure 4. Heating half processing. 
In Figure $4 b$, the histogram of the image in Figure $4 a$ is provided after normalization. The distribution of pixels has clear peaks. Each one groups those pixels that have an identical $a$ coefficient (number of occurrences). Therefore, it is possible to define ranges of $a$ coefficient values that corresponds to groups of pixels with the same heating tendency. The ranges are shown on the histogram using the vertical thick lines as borders.

\subsection{Cooling process}

The cooling half of the temporal evolution of the temperature for each pixel is a different tendency. In this case, up to three mechanism of heat transfers operate. Convection and radiation are clear but even conduction happens between the coil and the low thermal-diffusivity substrate. This process is much more complicated for modeling and, therefore, speed of the analysis reduces due to the time consuming algorithms. However, a cubic fitting is a good enough approximation. The analysis of the different coefficients of such fitting leads to consider that defects could be better observed just by judging the image for the $d$ coefficient, although all of them could be valid. Figure 5 collects this image and its histogram which could be interpreted as in the heating process.

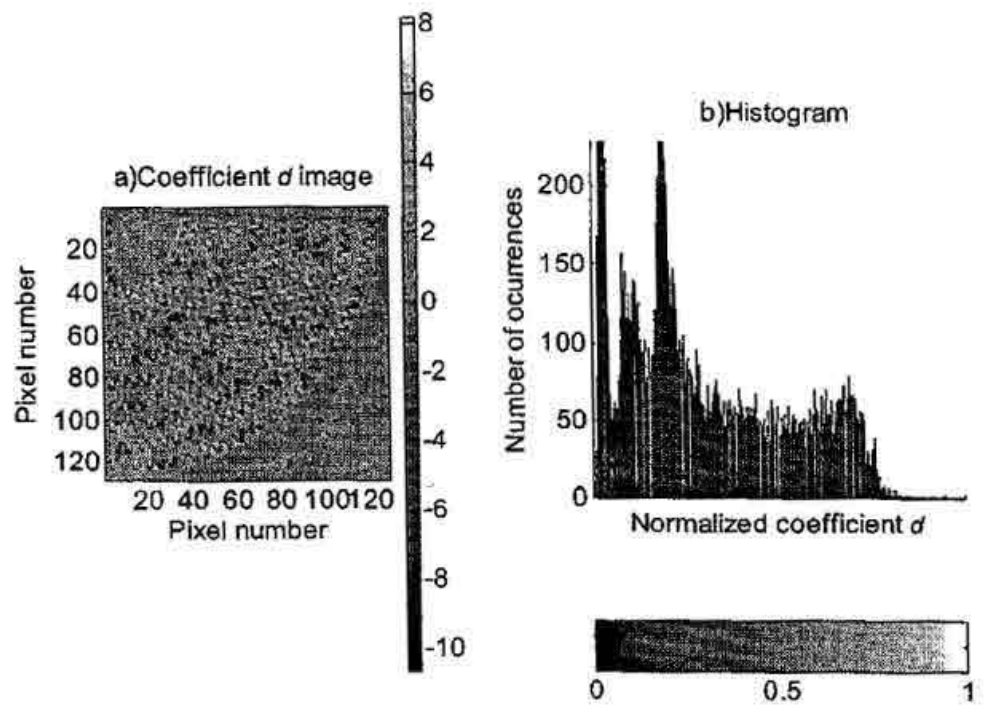

Figure 5. Cooling half processing.

\section{RESULTS DISCUSSION}

Dividing the histograms in shorter ranges of normalized coefficient values, several images can be obtained. For example, the range for the lowest normalized coefficients (range A) corresponds to pixels that are capturing the radiation from points of the background (independent on the radiant heater that remains approximately at same temperature during the excitation). The range $\mathrm{B}$ corresponds to the borders of the radiant heater in the image. The range $\mathrm{C}$ shows the pixels belonging to areas where the efficiency on quickly getting a high temperature is low (area corresponding to the neighborhoods of the inner and outer spires). The range D agrees with areas between coils, areas of substrate. The range $E$ relates to pixels referring to points near the heating element. The range $F$ relates to points on the wire and, finally, the range $\mathrm{G}$ shows the points with an atypical history.

Table 1 collects the images for the heating and cooling halves for each range. Observing these images the detection of the defects is clear as it corresponds to mismatches or lacks of continuity in each one of the represented areas. 


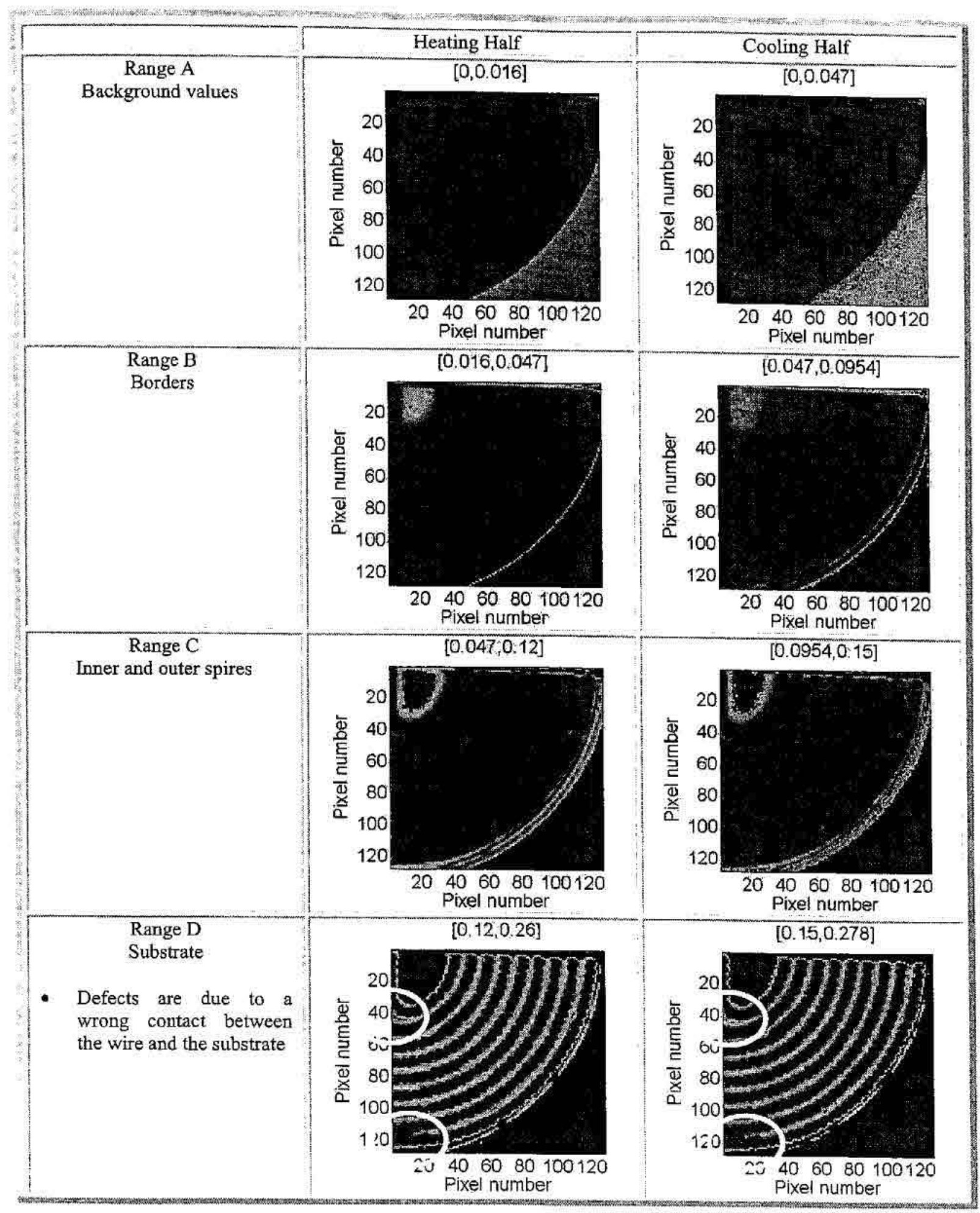




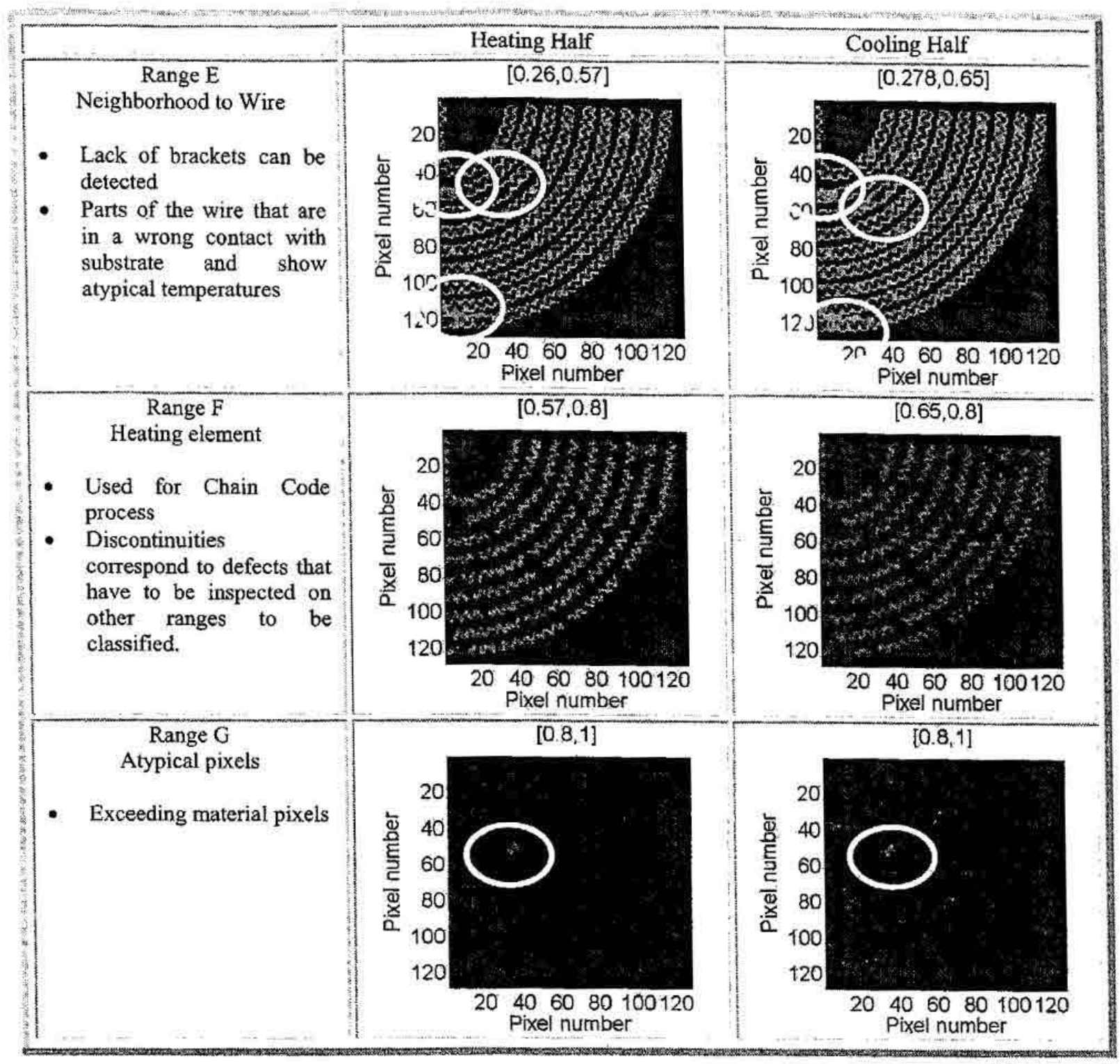

Table 1. Images for each one of the considered ranges. The defects are circled. Range F helps to determine the pattern of the coil. All the defects can be identified there by presenting a discontinuity on the coil pattern. Values over each graph show the range of normalized coefficient that each image represents.

\subsection{Location and classification of defects}

Further research has been carried out using morphing tools for the "virtual" generation of the pattern followed by the $\mathrm{coil}^{3}$. A model developed in Matlab ${ }^{\mathrm{TM}}$ using morphological operations on the image of the range $\mathrm{F}$ leads to the creation of binary images with pattern recognition. The "chain coding" was used in order to follow the wire drawn in that image and so the defects are located as these are the pixels missed to obtain a clearly defined pattern of the wire. The pixels of interest (those where defects are located) will be check in the rest of range images where they show variations from the common behavior. 
The possible defects can be classified in: i) lack of supporting brackets; ii) defects originated by a deficiency in the heating material; iii) defects due to excess of heating material (links, overlapping segments belonging to different wires, etc); and iv) parts of the heating elements which are in wrong contact (non-contact or semi-buried) with the substrate. The first group will be detected in images of range $\mathrm{E}$. The brackets are hot points linking both faces of the wire and are shown each a certain distance. Deficiencies on heating material will be shown on range $\mathrm{E}$ as colder areas as the faces of the wire will be in a lower temperature. On the other hand, links and overlapping will correspond to the hottest spots of the images and are the only ones to be seen on range $\mathrm{G}$. The deficit of substrate underneath the heating element (because the wire is without contact) allows to transfer more heat to the air by convection. Therefore, it creates a colder area as well as a blur region in the substrate area of the image in range D. Finally, the semi-buried heating elements are revealed by hot areas with some amount of blurring in the same substrate image in range D.

With this simple analysis of the provided images, defects can be detected, located and classified.

\section{CONCLUSIONS}

The objective of this work was to present a quality control process to be used on an industry dedicated to radiant heater manufacturing. Infrared Thermography has been used and its effectiveness as a NDT\&E technique for this niche of application has been demonstrated. After a short pulse excitation of $0.1 \mathrm{sc}$ (other values could be set but should provide enough energy), the recording of thermal images during the heating and cooling tendencies provides significant information for the defects that can appear on the production line. Due to such a quasi instantaneous excitation, a fast infrared camera was part of the setup. In addition to a simple processing, an inspection process has been established to detect and classify defects on the heaters in just a few seconds. In particular, the range of real and artificial defects in a commercial radiant heater includes: lack of supporting brackets; defects originated by a deficiency in the heating material; defects from an excess of heating material; and parts of the heating elements which are in wrong contact (noncontact or semi-buried) with the substrate. All of them have been successfully detected by the fast, non-contact and safe quality control process herein presented.

\section{ACKNOWLEDGMENTS}

The Spanish Science and Technology Minister under project SiRAS TEC2004-05936-C02-02 have supported this work. The authors also thank Maarten Mulder for the discussion and help provided in the development of this paper as well as Josu Duoandikoetxea for his practical support.

\section{REFERENCES}

\footnotetext{
${ }^{1} \mathrm{X}$. Maldague, Theory and Practice of Infrared Thermography for Nondestructive Evaluation, New York, John Wiley pub., 2001, 684p.

${ }^{2}$ D.A. González, F.J. Madruga, M.A. Quintela and J.M. López-Higuera, Defect assessment on radiant heaters using infrared thermography, NDT \& E International, Volume 38, Issue 6, September 2005, Pages 428-432

3 A. Escalera Hueso, Visión por computador. Fundamentos y métodos, Prentice Hall, Pearson Education, 2001, 274p.

${ }^{4} \mathrm{H}$. Freeman., On the Encoding of Arbitrary Geometric Configurations, IRE Trans. Electronic Computers, Vol. EC-10, pp. $260-268,1961$.
} 